\title{
MULTIWIRE CORE FLUXGATE
}

\author{
Pavel Ripka ${ }^{\star 1}$, X.P. Li ${ }^{2}$, J. Fan ${ }^{2}$ \\ ${ }^{1}$ Faculty of Electrical Engineering, Czech Technical University in Prague \\ Technicka 2, 16627 Prague 6 - Czech Republic \\ ${ }^{2}$ Department of Mechanical Engineering, National University of Singapore \\ 9 Engineering Drive 1, Singapore 117576
}

*Corresponding author: Phone: +420 224 353945, Fax: +420 233339 929, e-mail: ripka@fel.cvit.cz

\begin{abstract}
Fluxgate sensors with core made of multiple microwires are analyzed. We show that any study on sensors based on microwires should begin with detailed characterization of the magnetic properties of the wire, as they are dramatically changing within centimeters. Increasing the number of wires increases the sensitivity and lowers the sensor noise; by proper grouping into serioparallel configuration the current and power consumption can be optimized. The achieved sensitivity of $30 \mathrm{mV} / \mu \mathrm{T}$ and noise level is $0.34 \mathrm{nT} / \sqrt{ } \mathrm{Hz} @ 1 \mathrm{~Hz}$ for double- wire core with dipolar interaction.
\end{abstract}

Keywords: orthogonal fluxgate, multiwires, magnetic wires

\section{INTRODUCTION}

Miniature precise low-power magnetic sensors are required for many applications including security, position sensing and compass [1]. High linearity, high temperature stability, low noise and low perming are needed. Although significant effort was invested into the development of new magnetic sensors, the only candidates for the precise applications are AMR sensors and fluxgates. Although some authors report magnetoresistors with $10 \mathrm{pT}$ noise, however this is white noise measured at frequencies higher than of $100 \mathrm{~Hz}$. The best noise values for magnetoresistors measured at $1 \mathrm{~Hz}$ are $200 \mathrm{pT} / \sqrt{\mathrm{Hz}}[2,3]$. AMR sensors have other precision limits [4] and often cannot meet mentioned requirements, research effort is therefore invested into the miniaturization of fluxgate sensors.

Thin low-cost PCB fluxgate sensor can reach temperature offset stability of $0.2 \mathrm{nT} / \mathrm{K}$ [5]. The sensor size can be further reduced by using microtechnology [6]. Serious problem is to find proper material for the core of miniature fluxgates. Sputtered and electrodeposited permalloy do not possess required parameters. Microfluxgate sensor with amorphous sputtered Co85Nb12Zr3 shows promising properties: coercivity of $0.03 \mathrm{Oe}$, and the permeability of 10000 was reported in [7].

Orthogonal fluxgates represent another approach to the miniaturization of fluxgate sensors. These almost forgotten sensors [8] reappeared recently; with wire core they have several advantages:

- low demagnetization factor which results in low crossfield error

- low power consumption

- no excitation coil is necessary since the excitation current flows directly through the wire

Sasada introduced fundamental mode of transverse fluxgate $[9,10]$. Some sensors have lower noise in this mode (which was not the case for our sensors), but in its simple mode fundamental-mode fluxgated exhibit high offset, which is changing with temperature [11]. Sasada offered in [11] an improved mode by periodical changing of the polarity of the excitation bias. This technique requires more complicated circuitry and it is in fact equivalent to fluxgate symmetrically excited by current waveform of complex shape. We may conclude that [11] confirmed that only deep saturation into both polarities guarantee operation with long-term offset stability. However in this study we consider only second-harmonic excitation, which for our sensors gave both higher sensitivity and lower perming compared to fundamental mode.

Single-wire transverse fluxgate sensors of 2nd harmonic type with amorphous cores were studied in [12]. Later it was shown that if the permeability tensor has non-zero off-diagonal component, sensor output can be detected from the voltage induced between the wire terminals, i.e. the fluxgate sensor has no coil at all [13].

Transverese fluxgate sensor can also be manufactured by planar technology [14]. 
One of the disadvantages of orthogonal fluxgate sensors with wire cores is relatively low sensitivity caused by low cross-sectional area [15]. Multiwire cores can solve this problem. In the first multiwire fluxgate sensor all wires were connected in parallel [16]. The authors of [16] observed non-linear increase of the sensitivity with increasing number of wires for very closely packed wire cores. They have observed only linear dependence for $5 \mathrm{~mm}$ distant wires. By replacing some of the magnetic wires with copper wires, they experimentally verified, that the effect cannot be explained by adding the influence of the excitation currents from the close neighboring wires. Also inactive magnetic wires do not significantly influence the sensitivity. The result was that the effect should be caused by ac magnetic interaction between the wires, but the origin of this interaction remained uncovered.

In this paper we further investigate that effect measuring also serial and antiserial combinations of the wires. Also more systematic study was needed as we found that the wire properties dramatically change within the same batch. We also considered magnetic interactions between the wires, which depend on their distance, and quality of the tuning circuit.

\section{THE EXPERIMENTS}

We have made systematic study on core made of glass-covered amorphous Co68.25Fe4.5Si12.25B15 wire. The wire is $22 \mu \mathrm{m}$ thick and the glass coating thickness is $2 \mu \mathrm{m}$.

First of all we investigated homogeneity of the magnetic properties along the wire length. For this we developed ambulatory measurement setup to measure longitudinal hysteresis curves and also DC field sensitivity in simulated fluxgate mode (2nd harmonic response to the DC field in the direction of the wire). We observed huge changes of these properties within $\mathrm{cm}$ distance (Fig. 1), which means that each wire should be tested individually before being used for the sensor core. Big changes in the magnetization characteristics can be explained by local residual stresses [17]. Fig. 2 shows how the sensor characteristics change at different points of the wire length. One of the main factors affecting the change of the shape are residual mechanical stresses in the wire and local regions with induced anisotropy causing spatially changing off-diagonal components of the permeability tensor. We assume that some of the stresses can be caused by imperfect glass layer.

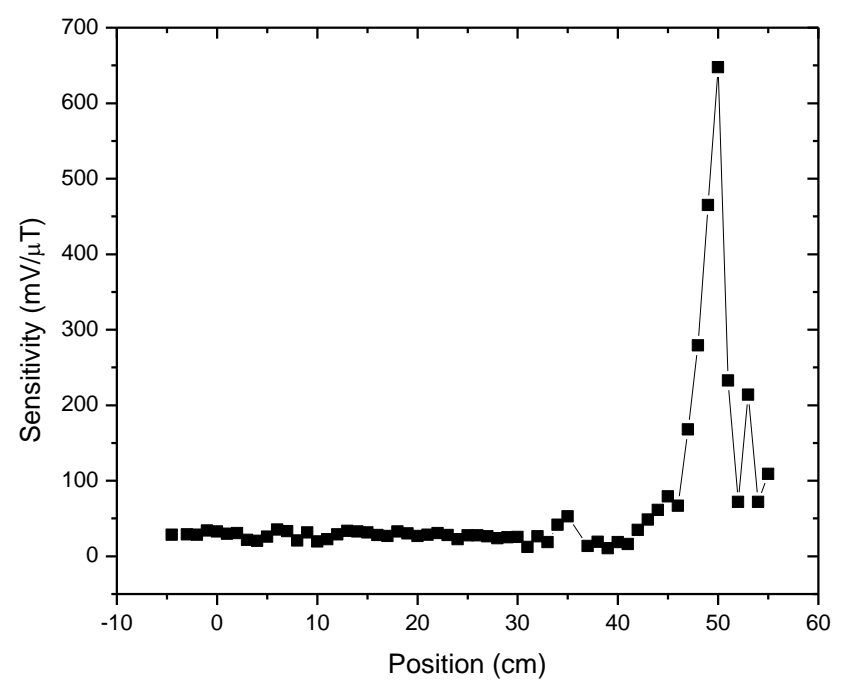

Figure 1. Sensitivity profile along the $60 \mathrm{~cm}$ section of the amorphous wire 


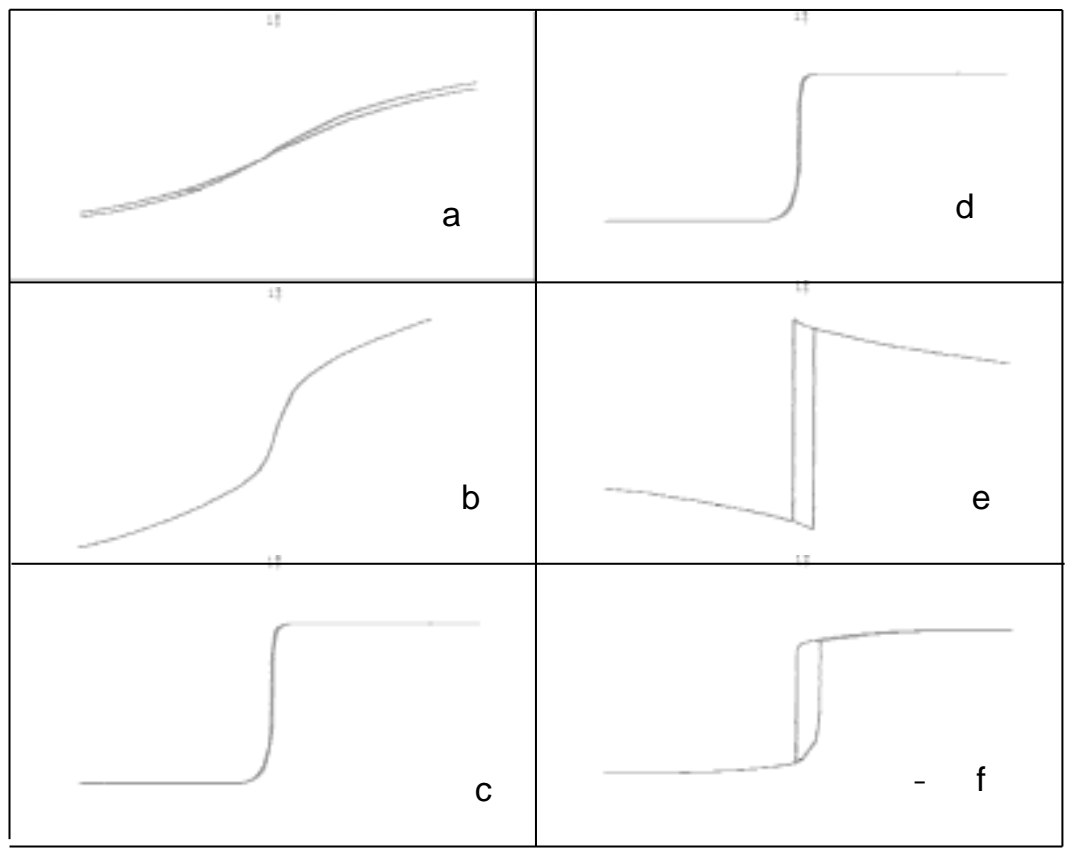

Figure 2. Sensor characteristics (second harmonics voltage versus longitudinal external field at different points of the wire from previous figure.

Later we cut $10 \mathrm{~mm}$ wire sections with similar characteristics and used them as the sensor cores. We changed the number of wires from 1 to 8 and their mutual distances (from direct contact with possible exchange coupling to $100 \mu \mathrm{m}$ resulting in fully dipolar interaction). The electrical connection was either parallel, serial, antiserial or combined serioparallel. In general, parallel connected wires require large amplitude for the excitation current, which is very unpractical. On the other hand serial connection of higher number of wire cores may requirev high excitation voltage. It is possible to use current transformer in the excitation circuit, but we found more simple solution - group wires to serioparallel combinations to optimize the impedance to the excitation source.

We also investigated the frequency dependence of the sensitivity and compared it with models. The pickup coil always had 1015 turns. The excitation frequency was always tuned for maximum sensitivity - the resonance frequency depends on the parasitic capacitance and mean inductance of the pick-up coil (which depends on the excitation parameters). The typical tuning curve is shown in Fig. 3.

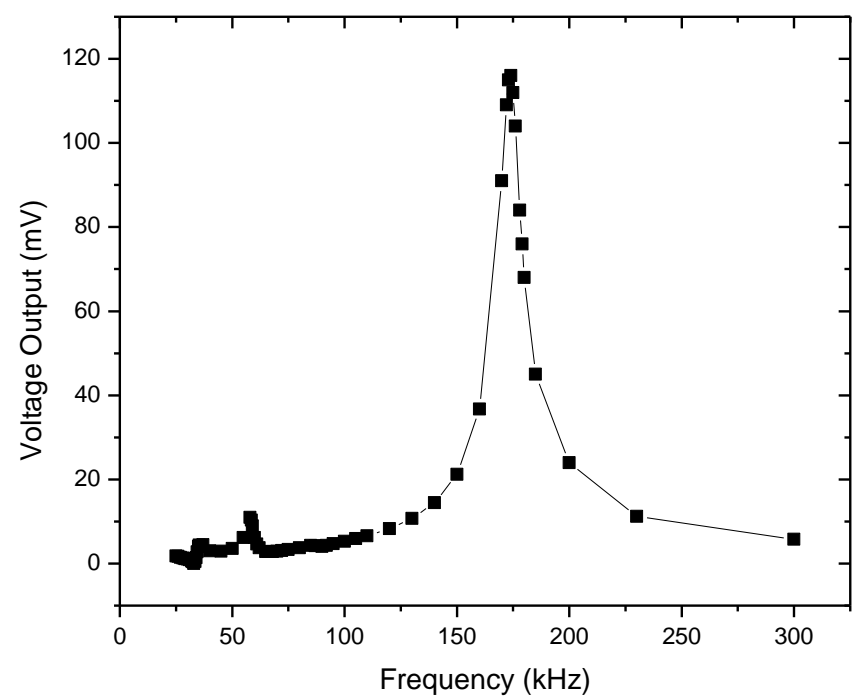

Figure 3. Frequency dependency of sensitivity: lexc $=20 \mathrm{~mA} \mathrm{rms}$, external field $=8.6 \mathrm{uT}$ 
An example of the measured sensitivity curves is shown in Fig. 4: two wires (T1A and T1B were used individually (single-wire cores) or closely together, either in parallel or antiserial connection. The results show that the sensitivities for double cores are more than twice the sensitivity of single-wire sensors. We explain this fact by increasing of the quality of the tuning circuit due to larger crosssection of inserted ferromagnetic material. The frequency characteristics of the sensitivity shows that with the exception of highest frequencies the sensitivity of parallel and antiserially connected cores are the same. The important advantage of antiserial connection is that the amplitude of spurious voltage at the excitation frequency is lower and thus the signal processing is much easier. Disadvantage of parallel connection is that it requires double excitation current.

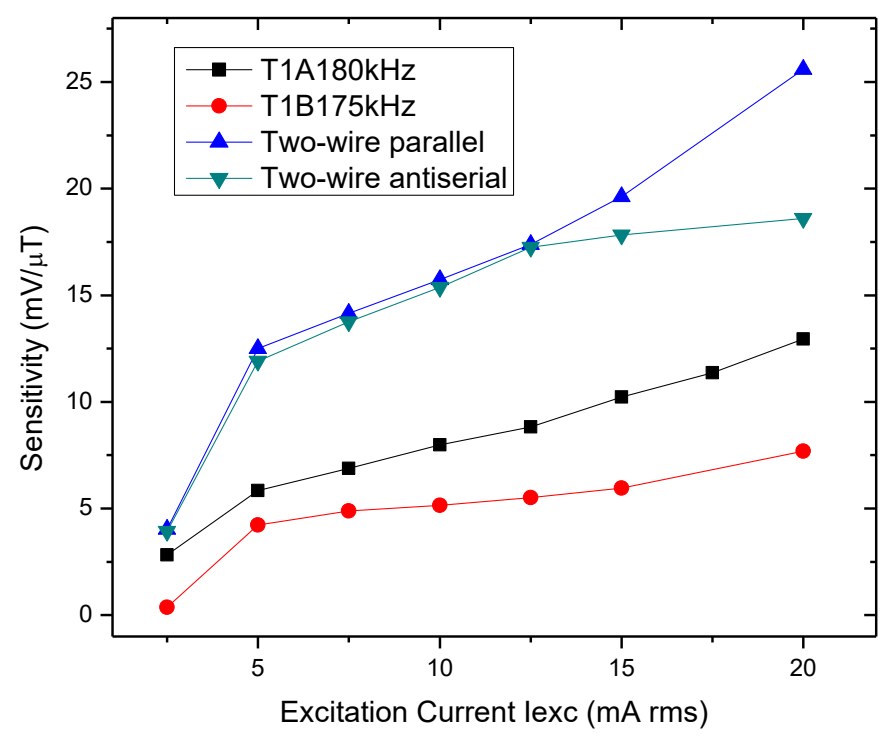

Figure 4: Sensitivity using $T 1 A$ and $T 1 B$ as cores for tuned fluxgate sensor: single-wire versus closely mounted doublewires (either connected in parallel or antiserially). The indicated excitation current flows through each wire.

We have measured the noise and sensitivity of our sensor demonstrators also for changing distance between the wires. The lowest noise of $0.34 \mathrm{nT} / \sqrt{\mathrm{Hz}} @ 1 \mathrm{~Hz}(1.2 \mathrm{nT} \mathrm{rms}$ in $30 \mathrm{mHz} . .10 \mathrm{~Hz}$ range $)$ was achieved for core made of antiserially connected wires with dipolar interaction (Fig. 5). The noise in the time domain and short-term (10-minute) offset stability at constant temperature are shown in Fig. 6. Distance between the wires was approximately $100 \mu \mathrm{m}$ and excitation current was $20 \mathrm{~mA} \mathrm{rms}$. With the excitation current reduced to $10 \mathrm{~mA}$ the noise increased to $0.52 \mathrm{TnT} / \sqrt{\mathrm{Hz}} @ 1 \mathrm{~Hz}$. These are values competitive to AMR sensors. 


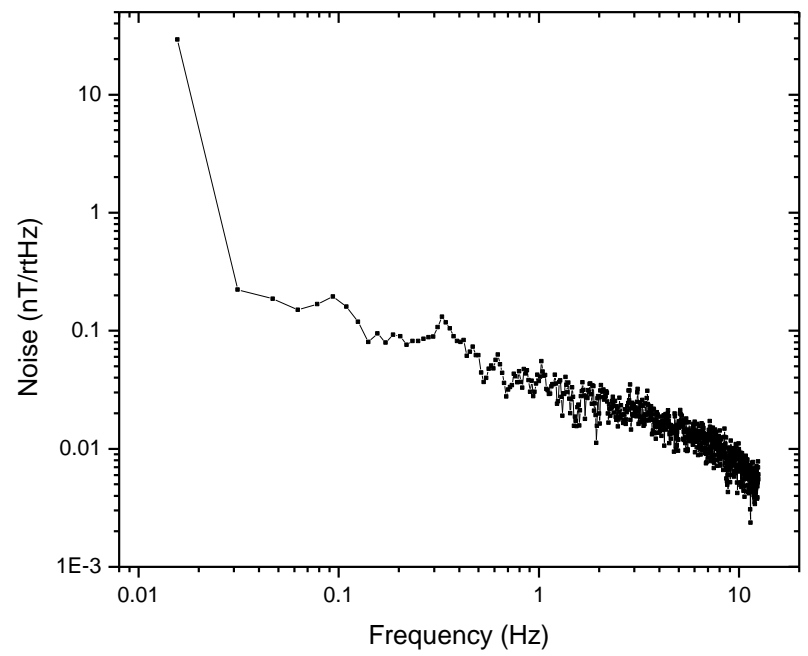

Figure 5. Noise for two-wire core with dipolar interaction excited antiserially

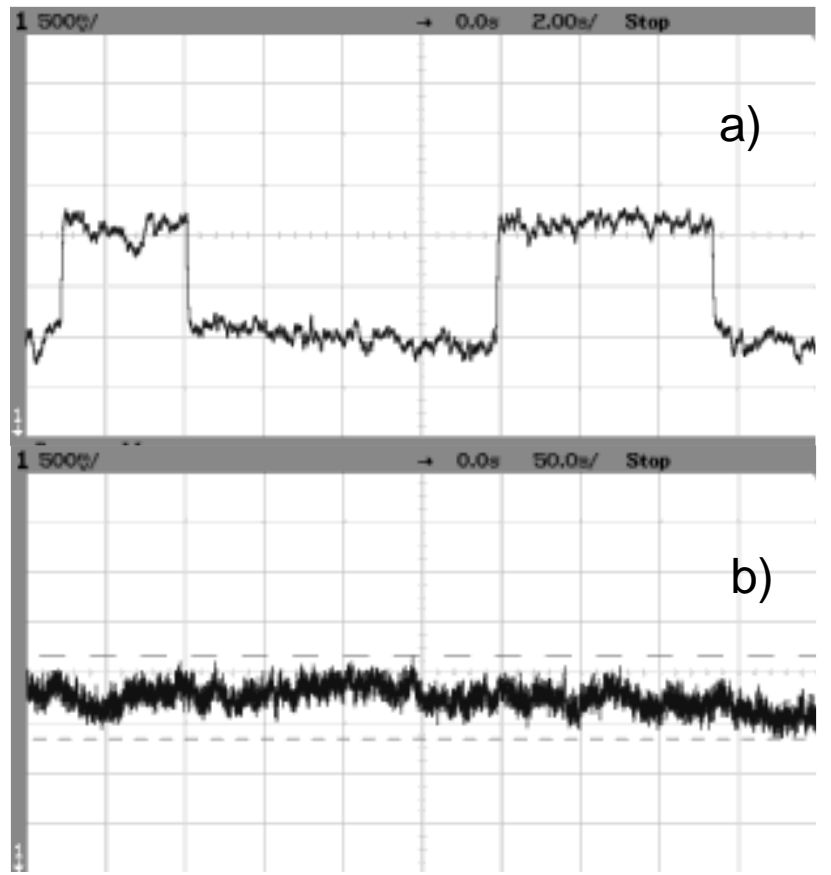

Figure 6. Noise of the same sensor in the time domain:

a) response to $10 \mathrm{nT}$ field step, b) 10-minute stability (same y scale $5 \mathrm{nT} / \mathrm{div}$ )

The sensitivity of the same sensor as a function of the excitation current rms value is shown in Fig. 6 . The sensitivity was calculated over the range of $34 \mathrm{~A} / \mathrm{m}$ (lower curve) and $7 \mathrm{~A} / \mathrm{m}$ (upper curve). The results show that even with the maximum value of the excitation current the core is still not saturated. We also observed that in this case the noise decreases with increasing of the excitation level proportionally to the increase of the low-field sensitivity. 


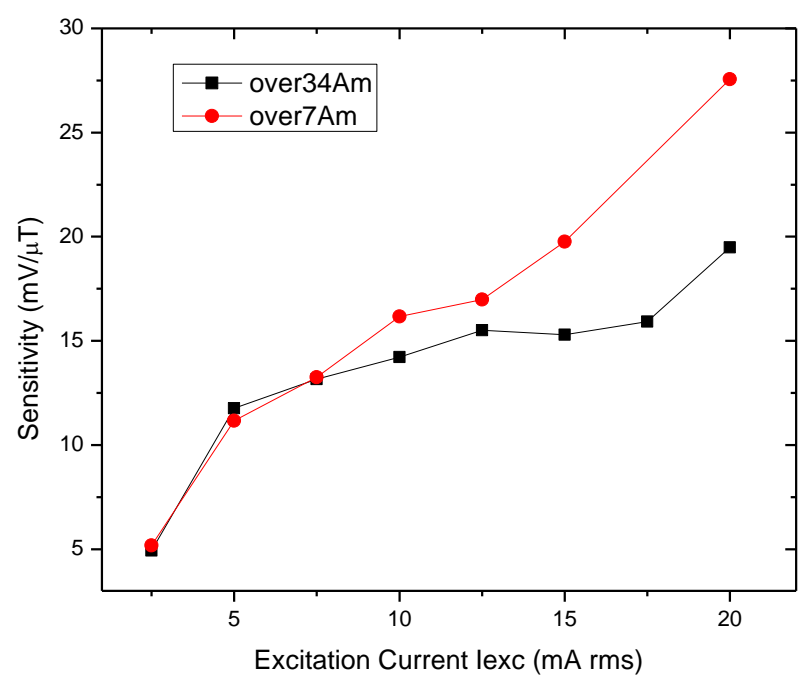

Figure 7. Sensitivity over two field ranges for core made of two antiserially connected wires with $100 \mu$ m distance

\section{CONCLUSIONS}

The sensitivity of orthogonal fluxgate sensors with wire core can be increased by using multiple wire core. Sensitivity of such system depends on the demagnetization factor, but it is also strongly dependent on the quality factor of the tuned output circuit. In our experiments presented in this paper we always tuned the pick-up coil by changing the excitation frequency, as this leads to best sensitivity and lowest noise. We have shown that both serial and antiserial connection of the wires is possible and the necessary excitation current is reduced compared to paralell connection. Moreover the antiserial configuration lowers the spurious voltage at the sensor output. The limitation for the number of serially connected wires is the generator voltage. The achieved noise level of $0.34 \mathrm{nT} / \sqrt{\mathrm{Hz}} @ 1 \mathrm{~Hz}$ shows that these sensors can be a serious competitor to AMR magnetoresistors. The achieved $5 \mathrm{nT}$ stability is sufficient for precise compass.

\section{REFERENCES}

[1] Vcelak J., V. Petrucha, and P. Kašpar: Electronic Compass with Miniature Fluxgate Sensors, Sensor Lett. 5, 279-282 (2007), doi:10.1016/j.jmmm.2008.04.176

[2] Stutzke NA, Russek SE, Pappas DP, et al.: Low-frequency noise measurements on commercial magnetoresistive magnetic field sensors JOURNAL OF APPLIED PHYSICS 97 (10): Art. No. 10Q107 Part 3 MAY 152005

[3] Zimmermann E., A. Verweerd, W. Glaas, A. Tillmann, A. Kemna, An AMR sensor-based measurement system for magnetoelectrical resistivity tomography, IEEE Sensors Journal, Volume 5, Issue 2, April 2005, $233-241$

[4] A. Bertoldi, Bassi D., Ricci L. et al.: Magnetoresistive magnetometer with improved bandwidth and response characteristics Rev. Sci. Instrum. 76, 065106 (2005)

[5] J. Kubik , L. Pavel, P. Ripka, PCB racetrack fluxgate sensor with improved temperature stability, Sensors and Actuators A 130 (2006): 184-188

[6] Baschirotto A, Dallago E, Malcovati P, et al.: A fluxgate magnetic sensor: From PCB to microintegrated technology, IEEE Trans. Instrum. Meas. 56 (2007):

[7] Na, KW Yuan, JL Ji, JH Choi, Microfluxgate sensor with amorphous cobalt (Co-Nb-Zr) soft magnetic core for electronic compass, Journ. Appl. Phys. 99 (2006) VL 99 AR 08M306

[8] F. Primdahl, "The Fluxgate Mechanism, Part I: The Gating Curves of Parallel and Orthogonal Fluxgates", IEEE Trans. Magn., 6 (1970), pp. 376-383.

[9] Plotkin Anton, Eugene Paperno, Alexander Samohin, and Ichiro Sasada Compensation of the thermal drift in the sensitivity of fundamental-mode orthogonal fluxgates, J. Appl. Phys. 99, 08B305 (2006) 
[10] Goleman K, Sasada I: A triaxial orthogonal fluxgate magnetometer made of a single magnetic wire with three U-Shaped branches IEEE TRANS. MAGN. 43 (6): 2379-2381 2007

[11] Sasada I.,: Symmetric response obtained with an orthogonal fluxgate operating in fundamental mode, IEEE Trans. Magn., vol. 38, no. 5, pp. 3377-3379, May 2002.

[12] Z.J. Zhao, X.P. Li, H.L. Seet, X.B. Qian, P. Ripka: Comparative study of the sensing perfomance of orthogonal fluxgate sensors with different amorphous sensing elements, Sens. Act. A 136 (2007) pp. 90-94.

[13] M.Butta, P. Ripka, S. Atalay, F. E. Atalay, X.P. Li: Fluxgate effect in twisted magnetic wire, JMMM, in press, doi:10.1016/j.jmmm.2008.04.176

[14] Zorlu, O Kejik, P Popovic, RS: An orthogonal fluxgate-type magnetic microsensor with electroplated Permalloy core Sens. Act. A 135 (2007), pp 43-49

[15] P.Ripka (ed.): Magnetic sensors and magnetometer, Chapter 3, Artech 2001

[16] X.P. Li, J. Fan, J. Ding, X.B. Qian: Multi-core orthogonal fluxgate sensor, Journal of Magnetism and Magnetic Materials 300 (2006) e98-e103

[17] Bydzovsky J., L. Kraus, P.. Svec, M. Pasquale, M. Kollar: Strain sensors based on stressannealed Co96Fe2Vr7Si8B14 amorphous ribbons, Sens. Act. A 110 (2004), 82-86. 


\section{Pavel Ripka}

received an Engineering degree in 1984, a CSc (equivalent to $\mathrm{PhD}$ ) in 1989, Docent degree in 1996 and in 2002 Professor degree at the Czech Technical University, Prague, Czech Republic.

During 1991-1993, he was a visiting researcher at the Danish Technical University and during 2001 he was Marie Curie Fellow at the National University of Ireland, Galway. In 2005/6 he was a visiting researcher at the Institute for the Protection and the Security of the Citizen, Ispra, Italy. At present he is a head of the Department of Measurement, Faculty of Electrical Engineering, Czech Technical University. He is lecturing in Measurements, Engineering Magnetism and Sensors. His main research interests are Magnetic Sensors, especially Fluxgate and MR, eddy current detectors and their applications. He is a member of IEEE, Elektra society, Czech Metrological Society, Czech National IMEKO Committee, Eurosensors Steering Committee, and the advisory board of SMM conferences. In 2001 to 2005 he served as an Associated Editor of the IEEE Sensors Journal. He was a General Chairman of the Eurosensors XVI conference held in Prague in 2002. He is a author and co-author of 6 books, $>200$ papers and 5 patents.

\section{X.P. Li}

received his Ph.D. in 1991 from University of New South Wales, Australia. He joined the National University of Singapore in 1992, where he is currently an associate professor in the Department of Mechanical Engineering, Division of Bioengineering, and Graduate Programme of Bioengineering. His research current interests include neurosensors and nanomaching.

\section{J. Fan}

received his B. Eng. Degree from University of Science and Technology of China, in 2003 and currently is a Ph.D. student in the Department of Mechanical Engineering in National University of Singapore. His research interests include the micromagnetism, magnetic sensors and magnetometers, and magnetic sources detection system. 
Figure 1. Sensitivity profile along the $60 \mathrm{~cm}$ section of the amorphous wire

Figure 2. Sensor characteristics (second harmonics voltage versus longitudinal external field at different points of the wire from previous figure.

Figure 3. Frequency dependency of sensitivity: lexc $=20 \mathrm{~mA} \mathrm{rms}$, external field $=8.6 \mathrm{uT}$

Figure 4. Sensitivity using $\mathrm{T} 1 \mathrm{~A}$ and $\mathrm{T} 1 \mathrm{~B}$ as cores for tuned fluxgate sensor: single-wire versus closely mounted double-wires (either connected in parallel or antiserially). The indicated excitation current flows through each wire.

Figure 5. Noise for two-wire core with dipolar interaction excited antiserially

Figure 6. Noise of the same sensor in the time domain:

a) response to $10 \mathrm{nT}$ field step, b) 10-minute stability (same y scale $5 \mathrm{nT} / \mathrm{div}$ )

Figure 7. Sensitivity over two field ranges for core made of two antiserially connected wires with $100 \mu \mathrm{m}$ distance 